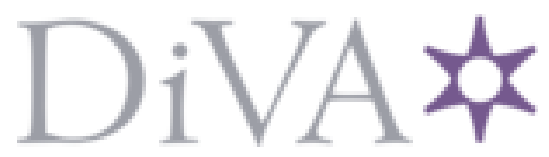

http://www.diva-portal.org

This is the published version of a paper presented at ALCOSSP 2013; 11th IFAC International Workshop on Adaptation and Learning in Control and Signal Processing; 3-5 July 2013; University of Caen Basse-Normandie, Caen, France.

Citation for the original published paper:

Pelckmans, K. (2013)

Randomized Gossip Algorithms for Achieving Consensus on the Majority Vote.

In: Proceedings of ALCOSSP 2013: 11th IFAC International Workshop on Adaptation and Learning in Control and Signal Processing

N.B. When citing this work, cite the original published paper.

Permanent link to this version:

http://urn.kb.se/resolve?urn=urn:nbn:se:uu:diva-213205 


\title{
Randomized Gossip Algorithms for Achieving Consensus on the Majority Vote
}

\author{
Kristiaan Pelckmans* \\ * Division of SysCon, Department of Information Technology, Uppsala \\ University, Sweden
}

\begin{abstract}
This paper studies a decentralized, randomized gossip algorithm for computing a majority vote amongst the binary decisions associated to $n$ nodes organized in a fixed, ad-hoc network. It is indicated how this problem can be reduced to computing the global average using a standard, randomized gossip algorithm. Then, we illustrate how the majority vote problem allows one to formulate individual stopping rules deciding when an individual node makes its final verdict. Finally, we will provide an illustration of how well the algorithm and associated stopping rule behaves.
\end{abstract}

Keywords: Randomize Gossip Algorithms, Consensus, Distributed Estimation.

\section{INTRODUCTION}

This paper proposes and studies decentralized algorithms for achieving consensus on the majority vote of $n$ different decision makers organized in a network. This elementary, distributed estimation task is used to explore new ideas in the context of randomized gossip algorithms. Such decentralized algorithms were largely stimulated by the recent Boyd et al. [2006] and earlier work of those authors, and borrows from results in a context of theoretical computer science (see e.g. Gilardoni and Clayton [1993], Karp et al. [2000]). This precise estimation task was considered in Bénézit et al. [2009]. The first novel contribution of this paper is a proposal for an individual stopping rule. That is, after how many iterations is it safe for an individual node to make its final decision (a node's verdict on its estimate of the global majority vote)? A stopping rule is proposed based on a node's estimate, and it is shown that (i) this rule will with high probability not yield false verdicts, and (ii) that nodes will achieve such verdict in a bounded number of iterations unless the global vote is (almost) tied. Secondly, we compare this scheme to the results given in Bénézit et al. [2009, 2011]. This work should be seen in the context of the study of the authors to engineer new decentralized estimation algorithms for non-standard computation tasks, already resulting in Pelckmans and Suykens [2009].

In general, a voting system or an electoral system is a protocol regulating how different voters arrive at a consensus. In the context of this paper, we will abstract this setup as a communication network amongst $n$ nodes where (i) each node makes a binary decision, (ii) the voting system collects, counts and (iii) distributes the majority vote amongst all nodes. Traditionally, it is implemented as a centralized or hierarchical system where a central unit processes all votes and sends out the global decision. It might be argued that such a system is not always realistic to implement, or is often prone to failure or malicious influence.
Algorithms for achieving consensus on the majority vote are of direct relevance in different scientific areas. We note its use in (1) theoretical computer science. Particularly, in the design and study of wireless Sensor Networks (WSNs) decentralized majority voting schemes can be of direct relevance. A principal incentive for studying fully decentralized algorithms in this context is to improve reliability and robustness against failure Malkhi and Reiter [1998] of part of the system, while avoiding the bottleneck of a central instance. A practical application might be found in the context of initiating a protocol for communication in a WSN: depending on the actual use (i.e. location, conditions,...) of the sensors one might choose between two protocols favorable in different situations. A majority vote might then be employed to decide on the protocol which is globally most useful in the present situation. In the context of learning theory, the majority vote is found to be a quite useful technique for online learning, see the halving algorithm, e.g. the survey Cesa-Bianchi and Lugosi [2006]. (ii) In the context of applied statistics, the present work hints towards a theory of decentralized hypothesis testing (see e.g. Olfati-Saber et al. [2006]). In relation hereto, one might conceive of different applications in social and biological sciences, and the present algorithms might be good models for collective decision making capabilities as observed in e.g. flocks of animals.

This paper is organized as follows: After introducing some notation in the following subsection, we are set to describe the proposed algorithms as well as some theoretical characterizations in Section 2. Proofs are extended to Appendix $\mathrm{A}$ and $\mathrm{B}$ in order to improve readability.

\subsection{Some Notation}

The following notation is used throughout the paper. Let random quantities be represented by capitals, and let deterministic elements as constants, functions, variables or vectors be represented as lower case letters. Matrices will be represented as bold face, e.g. $\mathbf{H}, \mathbf{L}, \mathbf{A}, \mathbf{W}, \ldots$ Let $\lambda_{i}(\mathbf{A})$ denote the $i$ th smallest eigenvalue of a matrix $\mathbf{A}$. 
Let $1_{n}$ denote the all-ones vector $1_{n}=(1, \ldots, 1)^{T}$ of size $n$, and let $e_{i}=(0 \ldots, 1, \ldots, 0)^{T}$ be the $i$ th unit vector. Let $|\cdot|$ denote the cardinality of a set, or dimensionality of a vector (when appropriate). We employ vector column notation, such that for all $\mathbf{H} \in \mathbb{R}^{n \times n}$ one has that $\mathbf{H} e_{i} \in \mathbb{R}^{n}$ yields a column with $n$ entries. We consider fixed graphs modeling the present communication network. Let $G=(V, E)$ be a loopless, positively weighted graph with $n$ vertices $V=\left\{v_{1}, \ldots, v_{n}\right\}$ and edges $\left\{e_{i j}\right\}$ with corresponding positive weights $\left\{a_{i j}\right\}$. Let the adjacency matrix $\mathbf{A} \in \mathbb{R}^{n \times n}$ collect those such that $\mathbf{A}_{i j}=a_{i j}$ for all $i, j=1, \ldots, n$. Let the degree $d_{i}$ of a node $v_{i}$ be defined as $\sum_{j=1}^{n} a_{i j}$, and let the degree matrix $\mathbf{D}_{\mathbf{A}} \in \mathbb{R}^{n \times n}$ be defined as $\mathbf{D}_{\mathbf{A}}=\operatorname{diag}\left(d_{1}, \ldots, d_{n}\right)$. The Laplacian $\mathbf{L}_{\mathbf{A}} \in \mathbb{R}^{n \times n}$ is defined as

$$
\mathbf{L}_{\mathbf{A}}=\mathbf{D}_{\mathbf{A}}-\mathbf{A}
$$

and let $\left\{\left(\lambda_{i}\left(\mathbf{L}_{\mathbf{A}}\right), \psi_{i}\right)\right\}_{i=1}^{n}$ denote the eigenvalues and corresponding eigenvectors $\psi_{i} \in \mathbb{R}^{n}$ of $\mathbf{L}_{\mathbf{A}}$. Note that by construction $\lambda_{1}\left(\mathbf{L}_{\mathbf{A}}\right)=0$ and $\psi_{1}=\frac{1_{n}}{\sqrt{n}}$. It will be seen that the convergence of the algorithms is a function of the second smallest eigenvalue of a matrix $\mathbf{W} \in \mathbb{R}^{n \times n}$ which is related to $\mathbf{L}_{\mathbf{A}}$, this measure is defined as follows (see Appendix A for an explanation)

Definition 1.

where

$$
\bar{\lambda} \triangleq \lambda_{2}(\mathbf{W})
$$

$$
\mathbf{W}=I_{n}-\frac{1}{n}\left(\mathbf{D}_{\mathbf{A}^{\prime}}-\mathbf{A}^{\prime}\right) \text {, }
$$

and where $\mathbf{A}^{\prime}=\mathbf{D}_{\mathbf{A}}^{-1} \mathbf{A}+\mathbf{A}^{T} \mathbf{D}_{\mathbf{A}}^{-1}$. Now $\mathbf{D}_{\mathbf{A}^{\prime}}=\operatorname{diag}\left(\mathbf{A}^{\prime} 1_{n}\right)$.

\section{SYNCHRONIZED RANDOMIZED GOSSIP ALGORITHMS}

\subsection{Voting by Average Gossip}

The first algorithm is a straightforward application of the synchronized gossip algorithm presented in (Boyd et al. [2006]). The key observation is that the majority vote amongst the votes $z_{1}, \ldots, z_{n}$ represented as binary values $\{-1,1\}$ made on the nodes $v_{1}, \ldots, v_{n}$ give a majority vote $\tilde{z} \in\{-1,0,1\}$ which can be written as

$$
\bar{v}=\operatorname{sign}\left(\sum_{i=1}^{n} z_{i}\right),
$$

where in case a tie occurs (or $\sum_{i=1}^{n} z_{i}=0$ ), a value of zero is returned. We denote the random variable containing the state of the $i$ th node at (right after) time-step $t$ as $Z_{i}^{(t)}$ for all $i=1, \ldots, n$ which takes values in the interval $[-1,1]$. The stochastic laws corresponding to this random variables are induced by the randomized algorithm.

Figure (1) gives an example trace (i.e. the evolution of the vector $Z^{(t)}=\left(Z_{1}^{(t)}, \ldots, Z_{n}^{(n)}\right) \in \mathbb{R}^{n}$ over time) of a single realization of the AG algorithm (1). The performance of this algorithm can be characterized using the techniques described in Boyd et al. [2006]. To state this result, we will use a notion of entropy of the majority vote, characterizing the excess in votes in favor of the majority vote:

Definition 2. (Binary Entropy). Let $\bar{z} \in[-1,1]$ be a number. Then the binary entropy may be defined as

$$
H(\bar{z})=-\frac{1}{2}\left(p \log _{2} p+(1-p) \log _{2}(1-p)\right),
$$

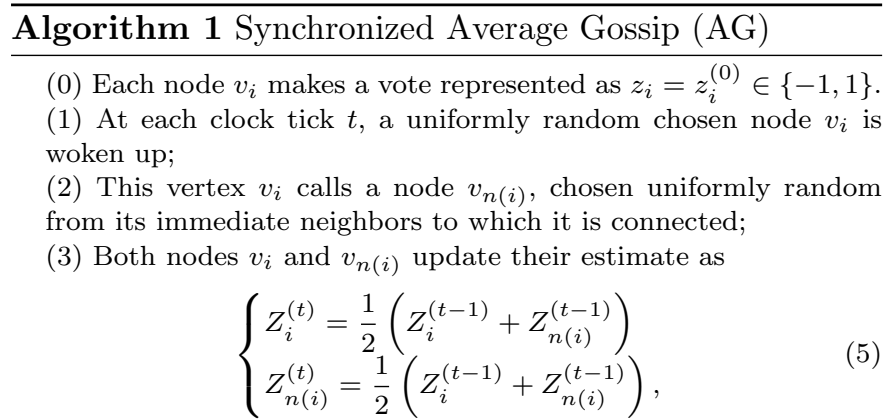

with $Z_{i}^{(t-1)}$ denoting the value as computed the last time the node $v_{i}$ was involved in an update, and with assignment to $Z_{i}^{(t)}$ at the time right after the update.

(4) The current estimate of the majority vote $\bar{v}$ at node $v_{i}$ is

$$
V_{i}^{(t)}=\operatorname{sign}\left(Z_{i}^{(t)}\right) .
$$

(5) Both nodes $v_{i}$ and $v_{n(i)}$ go to sleep again right behind time $t$; Steps (1)-(4) are iterated sufficiently long such that consensus is achieved on $\bar{v}$ with high probability.

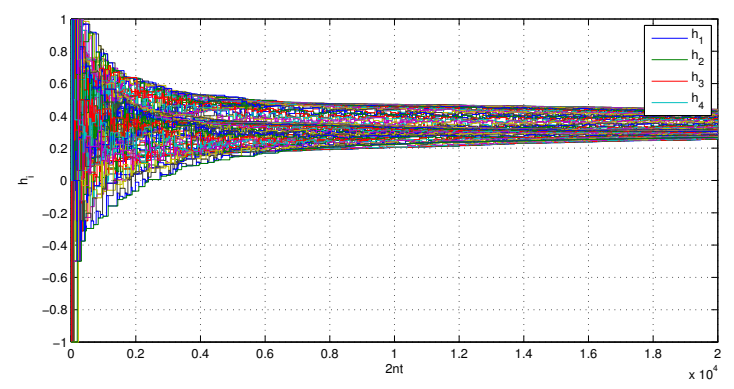

Fig. 1. Example trace of the $A G$ algorithm applied binary values implementing effectively a majority voting scheme. In this case of a random geometric graph with $n=200$ nodes it was enough to have (overall) roughly $t=4000$ iterations.

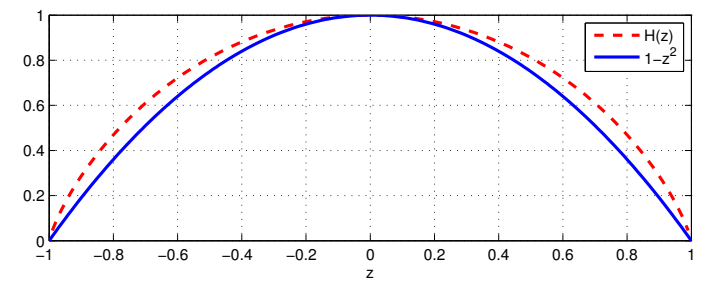

Fig. 2. The binary Entropy function as defined in eq. (7) $H(z)$ (dashed line); lowerbound $1-z^{2}$ (solid line).

where $p=\frac{1}{2}(\bar{z}+1)$.

Having a sample $\left\{z_{1}, \ldots, z_{n}\right\} \in\{-1,1\}^{n}$, with mean $\bar{z}=\frac{1}{n} \sum_{i=1}^{n} z_{i}$, Then $H(\bar{z})$ represents the 'excess' of the majority vote $\operatorname{sign}(\bar{z})$ (not necessarily adopting a stochastic setup). Furthermore, we have the inequality

$$
H(\bar{z}) \geq 1-\bar{z}^{2} \text {. }
$$

This inequality is illustrated in fig. (2).

Now the minimal amount of required iterations in $A G$ is bounded as follows.

Theorem 1. (Convergece of AG for Majority Votes). Let AG run for $t$ iterations, then any node $V_{i}=\operatorname{sign}\left(Z_{i}^{(t)}\right)$ achieves consensus on the majority vote $\bar{v}$ with probability exceeding 


$$
1-\left(\frac{4 \bar{\lambda}^{t} \sqrt{n}}{1-H(\bar{z})}\right) .
$$

Equivalently, for any confidence $\delta>0$ one achieves consensus in $t=O\left(-\ln \left(\frac{\delta}{\sqrt{n}}\right)-\ln (1-H(p))\right)$, where the constants depend on the network topology via the constant $\bar{\lambda}$.

In conclusion, it is 'easier' (it takes less iterations) to achieve consensus on a majority vote if the entropy of the majority vote is smaller.

This approach is powerful enough to implement quorumbased decision making. Here, we are interested whether a fraction of at least $\frac{1}{2}<\rho \leq 1$ of the nodes agree on the majority vote, and the decision is left undecided if this is not the case. This can be written in terms of the average $\bar{z}=\sum_{i=1}^{n} z_{i}$ as

$$
v_{\rho}=I(\bar{z}>\rho) \operatorname{sign}(\bar{z}) .
$$

\subsection{Gossip till Consensus}

While the mathematical properties of the AG gossip for voting are quite appealing, it might not be a very realistic voting model in practice. A main disadvantage is the rather implicit handling of the stopping rule - i.e. how long do we have to let the gossip scheme run to have guaranteed convergence to consensus at each node? While theorem 1 established global convergence to consensus in the synchronous scheme, the decision of achieving consensus is made in practical cases at each node (i.e. decentralized) as well. A simple way to achieve this is to define a fixed threshold $q$ for each node $v_{i}$, and compare the local estimates $Z_{i}^{(t)}$ with $q$ as well as the confidence currently achieved.

In general, we let the threshold be a positive function $q(\ldots) \in \mathbb{R}_{0}^{+}$which depends on the number of iteration $t$, the graph $G$ and a pre-specified confidence level $0<\delta<1$. The function $q$ converges to 0 as $\lim _{t \rightarrow \infty} q(\delta, G, t)=0$, and as such any node $v_{i}$ decides eventually on a final vote $V_{i}^{*}$ if $\hat{v} \neq 0$. We propose the following implementation of $q$ :

$$
q(\delta, G, t)=\frac{\pi^{2} \sqrt{n} \bar{\lambda}^{t} t^{2}}{3 \delta},
$$

which goes to zero when $\bar{\lambda}<1$. The modified algorithm is given in Alg. (2). It is important to note that (i) a node $v_{i}$ remains active in the gossip process, even after it has decided already on its verdict $V_{i}^{*}$. (ii) if a node is undecided at instant $t$ (i.e. if $\left|Z_{i}(t)\right| \leq q(\delta, G, t)$ ), it can mean that the algorithm hasn't run long enough, or that there is a global tie (i.e. $\bar{v}=0$ ).

\footnotetext{
Algorithm 2 Synchronized Average Gossip till Consensus (AGC)

(0) Let $\delta>0$ be a pre-specified confidence level, and initiate for the rest as in Alg. (1).

(1-4) as in Alg. (1)

(4b) A node finally decides on the vote $V_{i}^{*}=\operatorname{sign}\left(Z_{i}^{(t)}\right)$ (its 'verdict') when

$$
\left|Z_{i}^{(t)}\right| \geq q(\delta, G, t)
$$

(5) Repeat steps (1-4b) for at least $t$ as in eq. (14).
}

This algorithm possesses desirable convergence properties of the final votes $V_{i}^{*}$ for all nodes $v_{i}$ for $i=1, \ldots, n$. They essentially state that if running the algorithm long enough, one will have with high probability that (I) all nodes have a common verdict $V_{1}^{*}=\cdots=V_{n}^{*}=\bar{v}$ (no erroneous verdicts occur), and (II) the nodes remain undecided if the entropy is small or the algorithm hasn't run long enough.

Lemma 1. (Convergence of AGC). Fix a graph $G$ and a confidence level $\delta>0$. Then one has for all $t \geq 0$ and for all $i=1, \ldots, n$ that

$$
P\left(\bar{v} Z_{i}^{(t)} \leq-q(\delta, G, t)\right) \leq \delta .
$$

From this result, it follows immediately that if $t$ is large enough, i.e.

$$
t \geq \frac{\ln \left(\frac{4 n}{1-H(\bar{z})}\right)}{-2 \delta \ln (\bar{\lambda})} .
$$

Then one has with probability exceeding $1-\delta$ that for all nodes $v_{i} \in V$ that $\left|Z_{i}^{(t)}\right|>q(\delta, G, t)$, and each node made a verdict.

\subsection{Asynchronous Gossip}

An advantage of this randomized gossip scheme is that it can be modified quite straightforwardly to get rid of the central clock (i.e. formalized as the global variable $t$ ), synchronizing the operations. The central clock (or the global variable $t$ ) comes in in the algorithm at two points (i) in the function $q$, (ii) in the derivation of the sufficient running time $t^{\dagger}$. Rather than the central index $t$, we let each node be equipped with a clock indicating at each instant a unique $t_{s}>0$, monotonically increasing in time. The difference of the synchronous- and asynchronous gossip scheme (and hence between $t$ and $t_{s}$ ) is that the latter does not decides centrally who to wake up, and to allow a bijection between $t_{s}$ and the number of iterations $t$. The key idea then is that the central decision can be mimicked locally using a exponential distribution governing the activity (in continuous time) at each node. The consequence is (i) that two operations are performed at exactly the same time with probability zero; (ii) the activity pattern over the global network is uniformly distributed as in the synchronous case), (iii) the number of effective iterations $t$ can be estimated from $t_{s}$ and the number of nodes $n$. The analysis goes much along the lines of the previous subsection, and will be deferred to later.

\section{NUMERICAL COMPARISON}

Since the conception of this work, a competing algorithm was proposed and analyzed in Bénézit et al. [2009, 2011], see also Dimakis et al. [2010]. This section contrasts the current approach to this method.

The following experimental setup was implemented. We consider a ring consisting of $n=20$ nodes, each one connected to its two neighbors. Then we assign to the first $2 n / 3$ nodes a value $z_{i}=1$, and to the last $n / 3$ a value -1 (so the majority vote $\bar{v}=+1$ ). Then the algorithms are run in asynchronous mode, where a node is woken up by its own event, generated independently at each node, following an exponential distribution with intensity 1. That means that each unit of time, approximately $n$ 

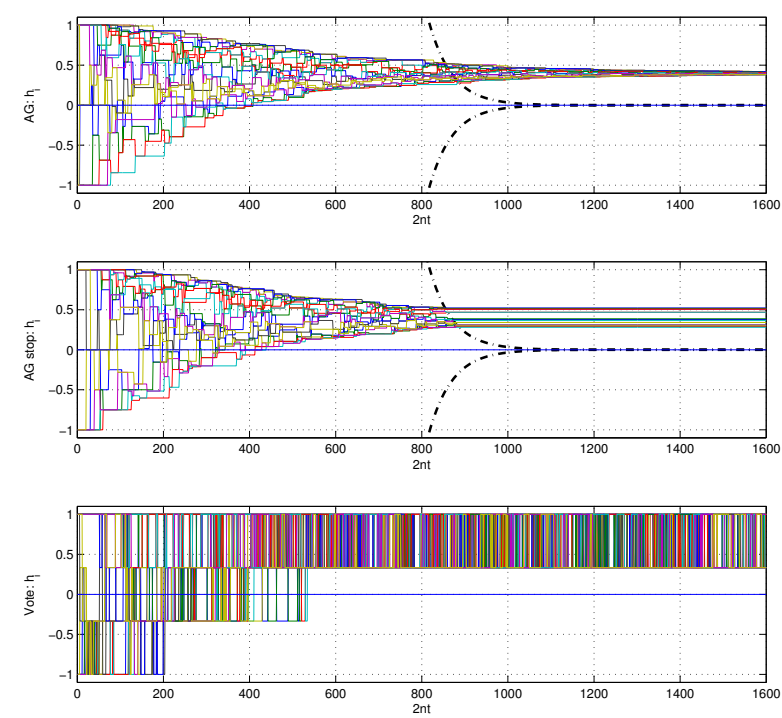

Fig. 3. Example traces of (a) the studied $A G$ algorithm, (b) the $A G$ algorithm where nodes which exceed $q(\delta, G, t)$ are removed from the algorithm. and (c) Benezit's voting scheme. The black lines in the first two plots show the value of $q(\delta, G, t)$ as in eq. (11). One sees that either scheme assigns the same sign ('consensus on the majority vote') to every node after around the same number of iterations (say $n \approx 600$ ). While the last scheme uses merely 4 different values to represent the state of a node, the $A G$ scheme also maintains a continuous value which can be interpreted as the 'margin' of a decision. This is the crucial ingredient for deriving a proper stopping rule. However, this comes at the price for the need of more refined hardware.

events (gossip operations) are performed. The results of three different algorithms are displayed in Figure (3).

(a) The asynchronous AG algorithm.

(b) The asynchronous AG algorithm, where nodes drop out of the active graph of the algorithms once they hit the threshold.

(c) The voter algorithm as detailed in Bénézit et al. [2009].

Note that (b) is not covered explicitly in the theorems, but since the choice of $q$ is uniform over all $n$ nodes, one can be reasonably sure that once the threshold is hit by a single node, the gossip scheme has converged enough to guarantee consensus. If consensus is reached, further $A G$ on the remaining nodes won't change the outcome. A few things can be observed from this simple experiment.

- The initial assignments of votes $z_{1}, \ldots, z_{n}$ was in some sense 'adversary', i.e. it is a difficult one to handle. In case of random $\{+1,-1\}$ assignments sampled from $B(p)$, the threshold $q$ becomes rather conservativel. This is illustrated in Figure (4).

- Experimental results indicate that the choice of $q$ in eq. (11) is not yet optimal. Specifically, experimental results suggest that it should have a $\log (n)$ dependence. On the other hand, the choice of the topology appears to affect the thresholding effect of the traces only through $\bar{\lambda}$ for similar topologies. This observations imply the need for a lower-bound on the choice of $q$.
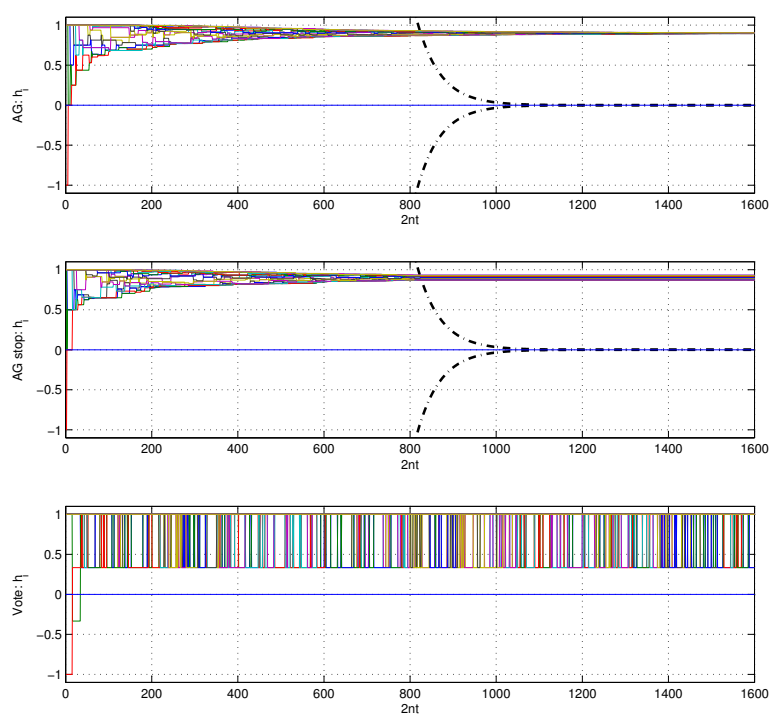

Fig. 4. Resulting trace of the algorithms using the same setup as before, but where the initial assignment of individual binary values is random (i.e. independent of the network topology). In this case we see that the bound $q(\delta, G, t)$ is very conservative. This is not surprising since the argument is worst case over the assignments $z_{1}, \ldots, z_{n}$.

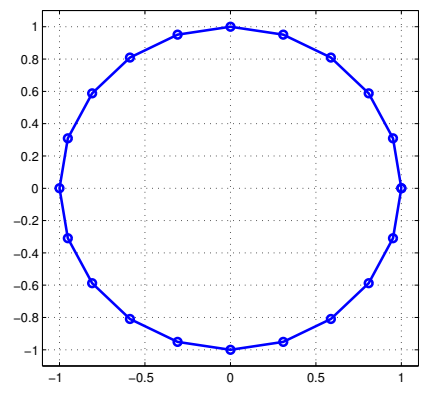

Fig. 5. The employed graph in the simulation, consisting of $n=20$ nodes arranged in a circle. For this topology, $\bar{\lambda}=0.9804$.

\section{DISCUSSION AND OPEN PROBLEMS}

This paper discusses some results we have for decentralized, randomized algorithms achieving consensus on the majority vote. This elementary task is studied in order to develop new insights in the design of randomized 'gossip' algorithms. Contributions included the design and analysis of individual stopping rules, and the numerical and theoretical study of those.

For the individual stopping times, it is an open question to us how to make the choice of $q$ less conservative, or if there exists a function $q^{\prime}$ which goes much faster to zero, bounding the probability of error to the same rate. A main open problem is how to extend ideas beyond the majority vote task. For example, it is not immediate how to design individual stopping rules if estimating continuous averages. Secondly, the device of random messages is found to be quite powerful, especially in situations were communication is subject to random failure, or communication 
packets are delayed. A formal analysis of the behavior of the present algorithm under such conditions is still open as yet.

\section{REFERENCES}

F. Bénézit, P. Thiran, and M. Vetterli. Interval consensus: from quantized gossip to voting. In Proceedings of ICASSP, volume 8, pages 3661 - 3664, 2009.

F. Bénézit, P. Thiran, and M. Vetterli. The distributed multiple voting problem. Selected Topics in Signal Processing, IEEE Journal of, (99):1-1, 2011.

S. Boyd, A. Ghosh, B. Prabhakar, and D. Shah. Randomized gossip algorithms. IEEE Transactions on Information Theory, 52(6):2508-2530, 2006.

N. Cesa-Bianchi and G. Lugosi. Prediction, Learning, and Games. Cambridge University Press, 2006.

F.R.K. Chung. Spectral Graph Theory. 1997.

A.G. Dimakis, S. Kar, J.M.F. Moura, M.G. Rabbat, and A. Scaglione. Gossip algorithms for distributed signal processing. Proceedings of the IEEE, 98(11):1847-1864, 2010.

G.L. Gilardoni and M.K. Clayton. On reaching a consensus using degroot's iterative pooling. The Annals of Statistics, 21(1):391-401, 1993.

R. Karp, C. Schindelhauer, S. Shenker, and B. Vocking. Randomized rumor spreading. In Annual Symposium on Foundations Of Computer Science, volume 41, pages 565-574, 2000.

D. Malkhi and M. Reiter. Byzantine quorum systems. Distributed Computing, 11(4):203-213, 1998.

R. Olfati-Saber, E. Franco, E. Frazzoli, and J. Shamma. Belief consensus and distributed hypothesis testing in sensor networks. Networked Embedded Sensing and Control, pages 169-182, 2006.

K. Pelckmans and J.A.K. Suykens. Gossip algorithms for computing u-statistics. In in Proc. of the 1st IFAC Workshop on Estimation and Control of Networked Systems (NecSys 2009), Venice,Italy, 2009.

\section{Appendix A. CONVERGENCE OF AG}

The following analysis goes along the lines as set out in Boyd et al. [2006], and convergence results for random walks. A specific gossip algorithm is characterized as a sequence of averaging operations, represented as matrices since $n<\infty$. Formally, at event time $t$, a node $v_{i}$ wakes up and calls $v_{j}$. They then exchange a piece of information. This can be written down in terms of a matrix $\mathbf{W}_{(i j)}$ where $\mathbf{W}_{(i j), k k}=1$ for all $k \neq i, j, \mathbf{W}_{(i j), i i}=$ $\mathbf{W}_{(i j), j j}=\mathbf{W}_{(i j), i j}=\mathbf{W}_{(i j), j i}=\frac{1}{2}$, and other entries zero, schematically

$$
\mathbf{W}_{(i j)}=\left[\begin{array}{ccccccc}
1 & & & & & & \\
& \ddots & & & & & \\
& & \frac{1}{2} & \cdots & \frac{1}{2} & & \\
& & \vdots & \ddots & \vdots & & \\
& \frac{1}{2} & \cdots & \frac{1}{2} & & \\
& & & & \ddots & \\
& & & & & 1
\end{array}\right] .
$$

As such, the algorithm makes at each iteration a random sample $W$ from the set $\left\{\mathbf{W}_{(i j)}, a_{i j}>0\right\}$ consistent with the underlying communication. The random draw is governed by the sampling protocol imposed in the algorithm: the expected matrix $\mathbf{W}=\mathbb{E}[W]$ can in this case be written as

$$
\mathbf{W}=\mathbb{E}\left[W^{(t)}\right]=\frac{1}{n} \sum_{i, j=1}^{n} \frac{a_{i j}}{\sum_{j=1}^{n} a_{i j}} \mathbf{W}_{(i j)} .
$$

As the matrix $\mathbf{W}$ is a linear combination of permutation matrices, $\mathbf{W}$ is a doubly stochastic matrix or $\mathbf{W} \geq 0$, $1_{n}^{T} \mathbf{W}=1_{n}^{T}$ and $\mathbf{W} 1_{n}=1_{n}$. Similar to the result given in theorem 3 of Boyd et al. [2006], one has that

Proposition 1. (The Expected Gossip Matrix W).

$$
\mathbf{W}=\mathbb{E}\left[W^{(t)}\right]=\frac{1}{n} \sum_{i j} \frac{a_{i j}}{d_{i}} \mathbf{W}_{(i j)}=I_{n}-\frac{1}{n}\left(\mathbf{D}_{\mathbf{A}^{\prime}}-\mathbf{A}^{\prime}\right) \text {. }
$$

where $\mathbf{A}^{\prime}=\mathbf{D}_{\mathbf{A}}^{-1} \mathbf{A}+\mathbf{A}^{T} \mathbf{D}_{\mathbf{A}}^{-1}$. Now $\mathbf{D}_{\mathbf{A}^{\prime}}=\operatorname{diag}\left(\mathbf{A}^{\prime} 1_{n}\right)$ denotes the degree matrix corresponding to this modified adjacency matrix.

Note that $\mathbf{W}$ is symmetric positive definite, and double stochastic regardless of the design of $\mathbf{A}$. In case of a regular, symmetric graph, we can relate the eigenvalues of $\mathbf{W}$ to the spectral properties of the underlying graph as follows

Proposition 2. (Expected Gossip Matrix). Let $G=(\mathbf{A}, V)$ be a given positively weighted graph with constant degree $k$, or $\sum_{j=1}^{n} a_{i j}=k$ for all $i=1, \ldots, n$, then one has

$$
\mathbf{W}=I_{n}-\frac{1}{k n}\left(\mathbf{L}(\mathbf{A})+\mathbf{L}\left(\mathbf{A}^{T}\right)\right) \text {. }
$$

Let $\left(\lambda_{i}\left(\mathbf{L}_{\mathbf{A}}\right), \psi_{i}^{\prime}\right)_{i}$ denote the eigenvalues and -vectors of the Laplacian $\mathbf{L}_{\mathbf{A}}$. If one has additionally that $\mathbf{A}=$ $\mathbf{A}^{T}$, then the eigenvalues and -vectors of $\mathbf{W}$ denoted as $\left(\lambda_{i}(\mathbf{W}), \phi_{i}\right)_{i}$ satisfy

$$
\lambda_{i}(\mathbf{W})=1-\frac{2}{k n} \lambda_{i}\left(\mathbf{L}_{\mathbf{A}}\right), \phi_{i}=\psi_{i}^{\prime}, \forall i=1, \ldots, n .
$$

Proof: $\quad$ This follows from rewriting eq. (A.3) as follows

$$
\begin{aligned}
\mathbf{W}=I_{n}-\frac{1}{n}\left(\mathbf{D}_{\mathbf{D}_{\mathbf{A}}^{-1} \mathbf{A}}\right. & \left.\left.-\mathbf{D}_{\mathbf{A}}^{-1} \mathbf{A}\right)\right) \\
& -\frac{1}{n}\left(\mathbf{D}_{\left.\mathbf{A}^{T} \mathbf{D}_{\mathbf{A}}^{-1}-\mathbf{A}^{T} \mathbf{D}_{\mathbf{A}}^{-1}\right),}\right.
\end{aligned}
$$

which is a consequence of the linearity of the degree matrix, i.e. $\mathbf{D}_{\mathbf{A}+\mathbf{A}^{\prime}}=\mathbf{D}_{\mathbf{A}}+\mathbf{D}_{\mathbf{A}^{\prime}}$ for all $\mathbf{A}, \mathbf{A}^{\prime} \in \mathbb{R}^{n \times n}$. When assuming a constant degree $k$, or $\mathbf{D}_{\mathbf{A}}=k I_{n}$, we can simplify (A.6) as follows

$$
\begin{array}{r}
\mathbf{W}=I_{n}-\frac{1}{k n}\left(\mathbf{D}_{\mathbf{A}}-\mathbf{A}\right)-\frac{1}{k n}\left(\mathbf{D}_{\mathbf{A}^{T}}-\mathbf{A}^{T}\right) \\
I_{n}-\frac{1}{k n} \mathbf{L}(\mathbf{A})-\frac{1}{k n} \mathbf{L}\left(\mathbf{A}^{T}\right) .
\end{array}
$$

If additionally, $\mathbf{A}=\mathbf{A}^{T}$, one can simplify further to $\mathbf{W}=I_{n}-\frac{2}{k n} \mathbf{L}(\mathbf{A})$ and the result follows.

Let us now derive the asymptotic bias after $t$ iterations. After $t$ iterations, let the estimates at the nodes be collected in a vector $Z^{(t)}=\left(Z_{1}^{(t)}, \ldots, Z_{n}^{(t)}\right) \in \mathbb{R}^{n}$, and let $z=Z^{(0)}$ contain the original measurements. 
Lemma 2. (Evolution of Variance). Consider a regular, connected communication graph. And let $\lambda_{2}$ be the second largest eigenvalue of $\mathbf{W}$ as in Proposition 2. After $t$ iterations of the Algorithm (1), one has

$$
\left\|\mathbb{E}\left[Z^{(t)}\right]-\bar{z} 1_{n}\right\|_{2} \leq\left\|z-\bar{z} 1_{n}\right\|_{2} \bar{\lambda}^{t}
$$

Proof: The algorithm AG is characterized as the difference equation

$$
Z^{(t)}=W^{(t)} Z^{(t-1)}=\prod_{s \leq t} W^{(s)} z
$$

where the product runs over all $t$ event times prior to $T$. Taking the expectation, and using independence of the different iterations of the algorithm we have that

$$
\mathbb{E}\left[Z^{(t)}\right]=\mathbf{W}^{t} z \text {. }
$$

Now a central insight for the analysis of random paths in weighted graphs (see e.g. Chung [1997], p.15), is that $\phi_{1}=\frac{1}{\sqrt{n}} 1_{n}$ and $\lambda_{1}=1$. It hence follows that

$\left\|\mathbb{E}\left[Z^{(t)}\right]-\left(\phi_{1} \phi_{1}\right)^{T} z\right\|_{2}=\left\|\sum_{i=2}^{n} \lambda_{i}^{t}\left(\phi_{i} \phi_{i}^{T}\right) z\right\|_{2} \leq\left\|z-\bar{z} 1_{n}\right\|_{2} \bar{\lambda}^{t}$,

with the inequality following from Cauchy-Schwarz. Furthermore, we have that $\bar{z}_{n} 1_{n}=\phi_{1} \phi_{1}^{T} z=\frac{1}{n} 1_{n \times 1} z$, (where the matrix $1_{n \times n} \in \mathbb{R}^{n \times n}$ has all one entries), and the result follows.

Convergence in expectation can be established using a slightly different formulation, and exploiting the matrix structure of $\mathbf{W}$.

Theorem 2. (Convergence of AverageGossip). After $t$ iterations of the Algorithm (1), one has for any $\epsilon>0$ that

$$
P\left(\left\|\bar{z} 1_{n}-Z^{(t)}\right\|_{2}^{2} \geq \epsilon\right) \leq \frac{\left\|z-\bar{z} 1_{n}\right\|_{2}^{2} \bar{\lambda}^{t}}{\epsilon}
$$

Proof: Then one can write as in the previous proof that

$$
\begin{aligned}
& \mathbb{E}\left[\left(\bar{z} 1_{n}-Z^{(t)}\right)^{T}\left(\bar{z} 1_{n}-Z^{(t)}\right)\right] \\
& \quad=\left(\bar{z} 1_{n}-z\right)^{T}(\mathbf{W} \mathbf{W})^{t}\left(\bar{z} 1_{n}-z\right) \\
& \quad=\left(\bar{z} 1_{n}-z\right)^{T} \mathbf{W}^{t}\left(\bar{z} 1_{n}-z\right) \leq \bar{\lambda}^{t}\left\|\bar{z} 1_{n}-z\right\|_{2}^{2}
\end{aligned}
$$

using respectively Cauchy-Shwarz' inequality and since $\mathbf{W}$ is idempotent.

The proof of Theorem 1 goes as follows. Proof: If the AG runs for a $t$ iterations, Lemma 2 states that for any $\epsilon>0$ one has

$$
P\left(\sup _{i}\left|Z_{i}^{(t)}-\bar{z}\right|^{2}>\epsilon\right) \leq P\left(\left\|Z^{(t)}-\bar{z} 1_{n}\right\|^{2}>\epsilon\right)<\frac{\bar{\lambda}^{t} n}{\epsilon}
$$

where we use the fact that $\left\|z-\bar{z} 1_{n}\right\| \leq \sqrt{n}$. Reshuffling terms gives

$$
P\left(\sup _{i}\left|Z_{i}^{(t)}-\bar{z}\right|^{2}>\bar{z}^{2}\right)<\frac{\bar{\lambda}^{t} \sqrt{n}}{\bar{z}^{2}}
$$

Now employing the lower-bound given in (8) yields the result.

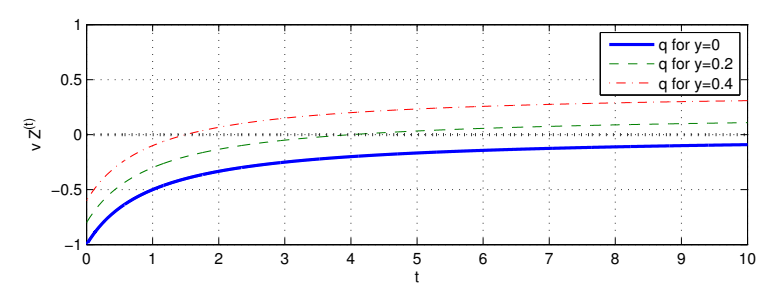

Fig. B.1. Illustration of the threshold $q(\delta, G, t)$ for values $t$ ranging from $0, \ldots, 10$ (curved solid line). The other 2 curved lines indicate the lowerbound for $\bar{z}=0.2$ and $\bar{z}=0.4$ respectively.

\section{Appendix B. INDIVIDUAL STOPPING RULES}

Lemma 2 is proven as follows. Proof: At first, fix $i=1, \ldots, n$ and $t \geq 0$, then

$$
\begin{aligned}
P\left(\bar{v} Z_{i}^{(t)}\right. & \leq-q(\delta, G, t)) \\
& \leq P\left(\left|Z_{i}^{(t)}-\bar{z}\right| \geq|\bar{z}|+q(\delta, G, t)\right) \\
& \leq P\left(\left|Z_{i}^{(t)}-\bar{z}\right| \geq q(\delta, G, t)\right) .
\end{aligned}
$$

Now, this value is bounded uniformly over $i, t$ by relating $q(\delta, G, t)$ to $\mathbb{E}\left\|Z_{i}^{(t)}-\bar{z} 1_{n}\right\|_{2}$ using the result of Theorem 2, one has $\mathbb{E}\left\|\bar{z} 1_{n}-Z^{(t)}\right\|^{2} \leq \bar{\lambda}^{t}\left\|\bar{z} 1_{n}-z\right\|_{2}^{2} \leq 2 \sqrt{n} \bar{\lambda}^{t}$ since $\left\|z-\bar{z} 1_{n}\right\|_{2} \leq 2 \sqrt{n}$ by construction. Hence

$$
h(t, \delta) \mathbb{E}\left\|Z_{i}^{(t)}-\bar{z} 1_{n}\right\|_{2} \leq h(t, \delta) 2 \sqrt{n} \bar{\lambda}^{t}=q(\delta, G, t),
$$

for $h(t, \delta)=t^{2} \frac{\pi^{2}}{6 \delta}>0$, as following from the definition of $q$. By application of Chebychev's inequality, it follows that

$$
\begin{aligned}
& P\left(\left\|Z^{(t)}-\bar{z} 1_{n}\right\|_{2} \geq q(\delta, G, t)\right) \\
& \quad \leq P\left(\left\|Z^{(t)}-\bar{z} 1_{n}\right\|_{2} \geq h(t, \delta) \mathbb{E}\left\|Z^{(t)}-\bar{z} 1_{n}\right\|_{2}\right) \\
& \leq \frac{1}{h(t, \delta)} .
\end{aligned}
$$

Now, since $\left\|Z^{(t)}-\bar{z} 1_{n}\right\|_{\infty} \leq\left\|Z^{(t)}-\bar{z} 1_{n}\right\|_{2}$, and one wants the bound to hold for all random values $Z^{(1)}, Z^{(2)}, \ldots, Z^{(t)}, \ldots$, one has that

$$
P\left(\sup _{i=1, \ldots, n} \sup _{t=1, \ldots}\left|Z_{i}^{(k)}-\bar{z}\right| \geq q(\delta, G, t)\right) \leq \sum_{t=1}^{\infty} \frac{1}{h(t, \delta)}
$$

by application of a Bonferroni correction. Consider the series $\sum_{t>0} \frac{1}{t^{2}}=\frac{\pi^{2}}{6}$, and recall that $h(t, \delta)=t^{2} \frac{\pi^{2}}{6 \delta}$, then

$$
\sum_{t=1}^{\infty} \frac{1}{h(t, \delta)}=\frac{6 \delta}{\pi^{2}} \sum_{t=1}^{\infty} \frac{1}{t^{2}}=\frac{\delta 6}{\pi^{2}} \frac{\pi^{2}}{6}=\delta
$$

After reshuffling terms $\left(\frac{\delta \pi^{2}}{12 \sqrt{n}}=\delta\right.$ or $\left.\delta=\frac{12 \delta^{\prime} \sqrt{n}}{\pi^{2}}\right)$ the result is proven. 